\title{
THE WHITE DWARF MASS AND ORBITAL PERIOD DISTRIBUTION IN ZERO-AGE CATACLYSMIC BINARIES
}

\author{
M. Politano and R. F. Webbink \\ University of Illinois at Urbana-Champaign
}

\section{INTRODUCTION}

A zero-age cataclysmic binary ( $Z A C B$ ) we define as a binary system at the onset of interaction as a cataclysmic variable. We present here the results of calculations of the distributions of white dwarf masses and of orbital periods in ZACBs, due to binaries present in a stellar population which has undergone continuous, constant star formation for $10^{10}$ years.

II. METHOD

Distributions of ZACBs were calculated for binaries formed $t$ years ago, for log $t=$ 7.4 (the youngest age at which viable ZACBs can form) to $\log t=10.0$ (the assumed age of the Galactic disk), in intervals of $\log t=0.1$. These distributions were then integrated over time to obtain the ZACB distribution for a constant rate of star formation. To compute the individual distributions for a given $t$, we require the density of systems forming (number of pre-cataclysmics forming per unit volume of orbital parameter space), $n_{f}(t)$, and the rates at which the radii of the secondary and of its Roche lobe are changing in time, $\dot{R}_{s}(t)$ and $\dot{R}_{L, s}(t)$, respectively. In calculating $n_{f}(t)$, we assume that the distribution of the orbital parameters in primordial (ZAMS) binaries may be written as the product of the distribution of masses of ZAMS stars (Miller and Scalo 1979), the distribution of mass ratios in ZAMS binaries (cf. Popova, et al., 1982), and the distribution of orbital periods in ZAMS binaries ( $A b t$ 1983). In transforming the the orbital parameters from progenitor (ZAMS) to offspring (ZACB) binaries, we assume that all of the orbital energy deposited into the envelope during the common envelope phase leading to $Z A C B$ formation goes into unbinding that envelope. $\dot{R}_{L, s}(t)$ is determined from orbital angular momentum loss rates due to gravitational radiation (Landau and Lifshitz 1951) and magnetic braking $(\gamma=2$ in Rappaport, Verbunt, and Joss 1983). We turn off magnetic braking if the secondary is completely convective. 


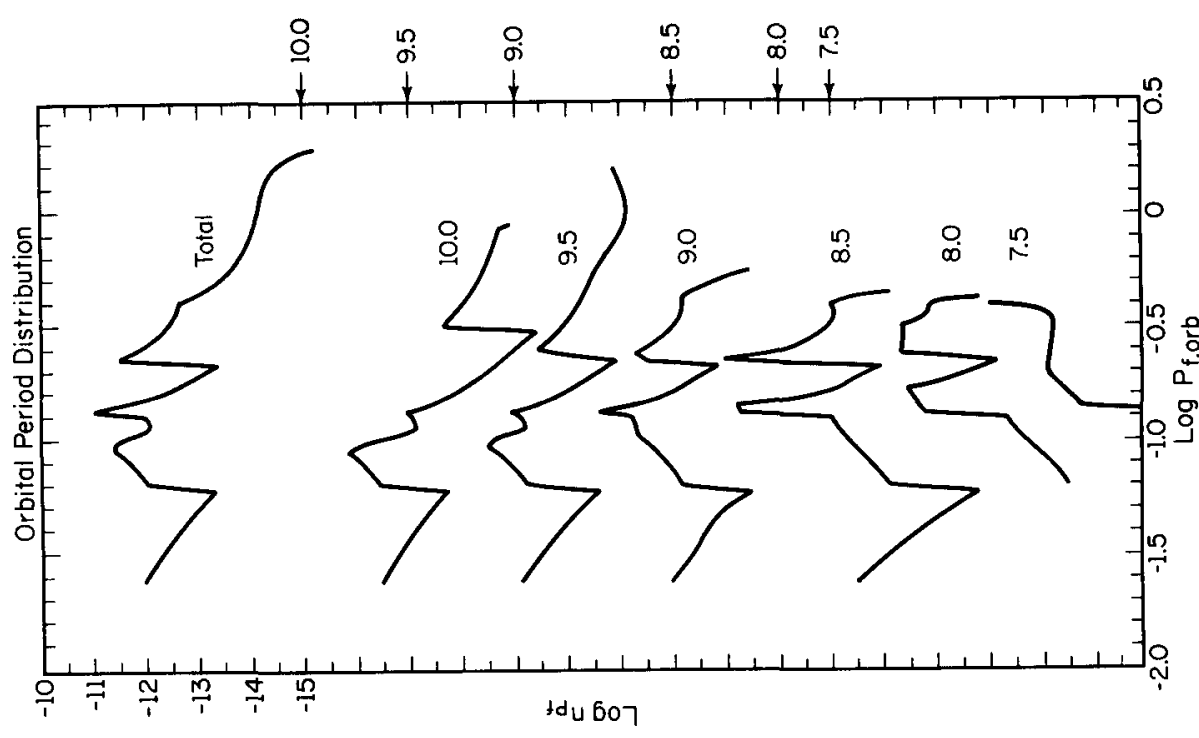

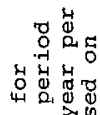

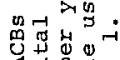

बून मु.

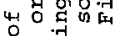
舟

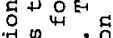

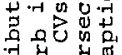

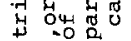
ती क्य 4 出

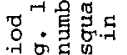

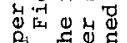
- 5 म 50

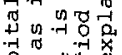

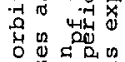
ב स

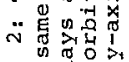

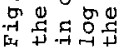

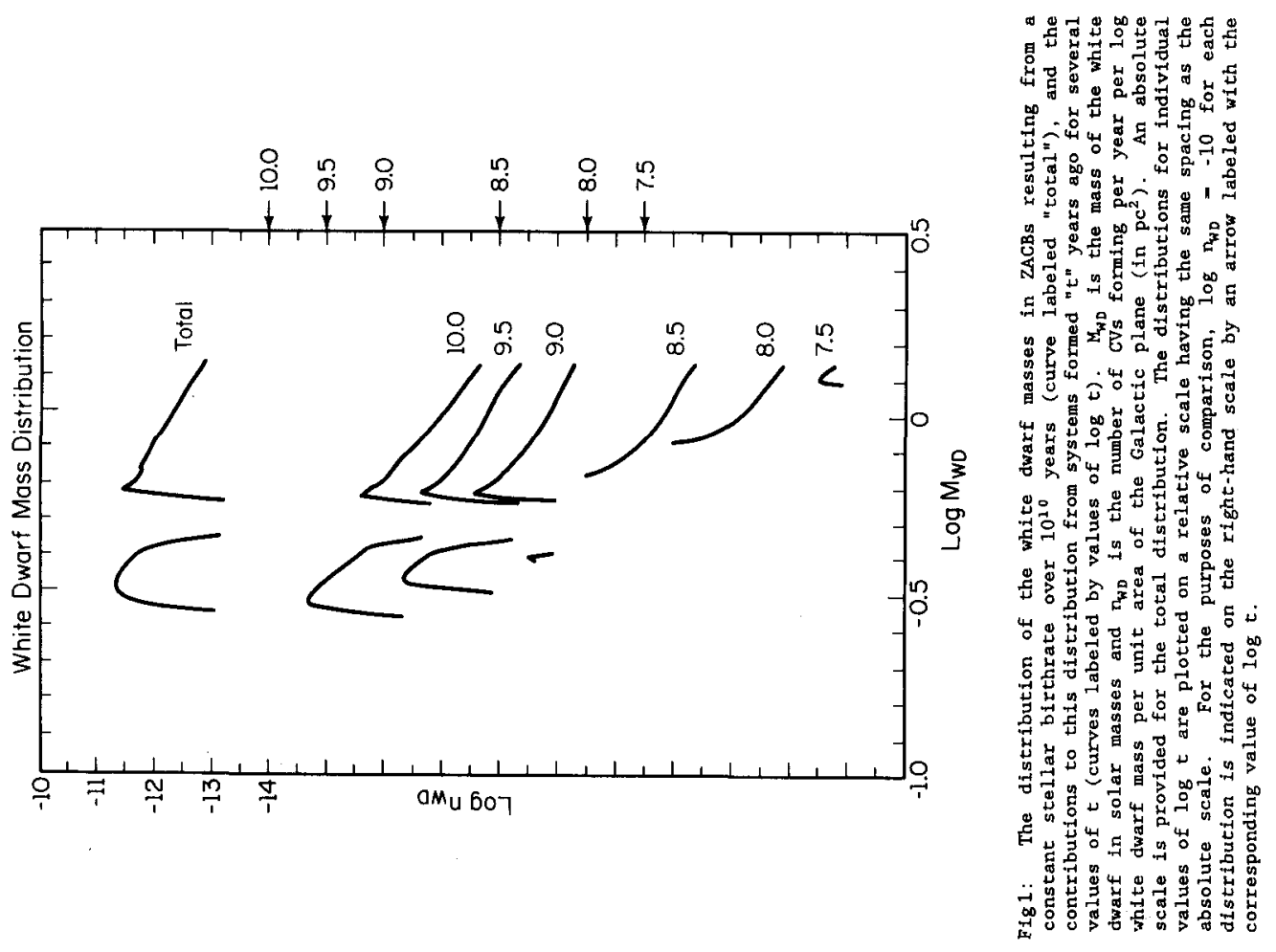


Figures 1 and 2 show the results of these calculations. Our principal conclusions may be summarized as follows:

- Cataclysmic variables (CVs) first appear in a stellar population at an approximate age $\log t=7.4$ years. This is the smallest age at which close binaries leave white dwarf remnants.

- The distribution of white dwarf masses in ZACBs is not sufficient to account for the observed high white dwarf masses in CVs, emphasizing the possible importance of selection effects (Ritter and Burkert 1986; see also Politano, Ritter, and Webbink, these proceedings).

- The orbital period distribution identifies four main subsets of ZACBs:

(1) short-period systems containing He white dwarfs (peak near $\log P=-1.1$ );

(2) systems with Co white dwarfs whose secondaries are convectively stable against rapid mass transfer to the white dwarf (peak near $\log P=-0.9$ );

(3) systems with co white dwarfs whose secondaries are radiatively stable against rapid mass transfer (peak near $\log P=-0.65$ ); and (4) long period systems with evolved secondaries (wing out to periods of roughly 2 days).

- The period distribution of ZACBs has a local minimum between 2 and 3 hours, due to the discontinuity in white dwarf masses between CVs containing He white dwarfs and systems containing Co white dwarfs (cf. Webbink 1979).

- CVs forming from binaries less than $10^{9}$ years old do not contain He white dwarfs.

- The formation rate of $\mathrm{CVs}$ is maximized when the nuclear time scale of the initial primary is comparable to the time scale for angular momentum loss during the pre $-\mathrm{CV}$ state. In this case, pre-CV systems are brought into contact roughly as fast as they are formed (emerge from the common envelope phase).

This research was supported in part by NSF grant AST 86-16992.

\section{REFERENCES}

Abt, H. 1983, Ann. Rev. Astron. Astrophys. 21, 343.

Landau, L., and Lifshitz, E. 1951, The Classical Theory of Fields, Addison-Wesley. Miller, G.E., and Scalo, J.M. 1979, Astrophys. J. Supp1., 41, 513.

Popova, E.I., Tutukov, A.V., and Yungelson, L.R. 1982, Astrophys. Space Sci., 88, 55 .

Rappaport, S., Verbunt, F., and Joss, P.C. 1983, Astrophys. J., $275,713$.

Ritter, H., and Burkert, A. 1986, Astron. Astrophys., 158, 161.

Webbink, R.F. 1979, in IAU Colloq. No. 53, White Dwarfs and Variable Degenerate Stars, H.M. Van Horn and V. Weidemann (eds.), Univ. of Rochester Press, p. 426. 